\title{
Collapsing magnetic instability to solar intermittent flux and anomalous viscosity in accretion disks
}

\author{
X. Q. Li and H. Zhang \\ Department of Physics, Nanjing Normal University, Nanjing 210097, PR China \\ Received 15 November 2001 / Accepted 24 April 2002

\begin{abstract}
It is shown that the transverse plasmon field is modulationally unstable in the Lyapunov sense, leading to a self-similar collapse of the magnetic flux. Such a collapsing magnetic instability is analyzed in both cases of magnetohydrodynamics and kinetic plasma physics, with their applications to solar intermittent flux and anomalous viscosity in accretion disks, respectively. In the first case, we find that the equilibrium between the ponderomotive force and the Lorentz force in a current sheet gives rise to a more spatially intermittent collapsing magnetic flux, very similar to a turbulent pattern; as a result, the $0.1 \mathrm{kG}$ flux cells with larger scales emerging at the surface from the solar interior become shredded and the flux is thereby contracted rather quickly to a small scale of the order of $100 \mathrm{~km}$ as well as concentrated into a 1-2 kG state. In the second case, based on Vlasov equations and Maxwell equations, the collapsing feature of the self-generated magnetic field from transverse plasmons is investigated on rather small scales of the motion or electric current in accretion disks; as the effects of the intermittent magnetic flux, an anomalous magnetic viscosity and an anomalous resistivity are indicated, with a different magnetic Prandtl number, which is not very sensitive to the temperature $T$.
\end{abstract}

Key words. accretion: accretion disks - magnetic fields - Sun: magnetic fields

\section{Introduction}

Magnetic field plays an important role in many fields of astrophysics. Its behavior is mainly set up by its interaction with its carrier: plasma. It is well known that a plasma is a system with a large number of degrees of freedom; in such a highly unstable plasma, the tendency for energy equipartition over the different possible degrees of freedom can produce turbulent waves, that is to say, plasmons are excited at a rather high level. In a plasma there is a transverse mode, with frequencies $\omega^{\mathrm{p}}$ which are nearly the frequency $\omega_{\mathrm{pe}}$ :

$\omega^{\mathrm{p}}=\omega_{\mathrm{pe}}+\frac{k^{2} c^{2}}{2 \omega_{\mathrm{pe}}} \quad\left(\omega_{\mathrm{pe}} \gg k c\right)$,

here $\omega_{\mathrm{pe}}=\frac{4 \pi \mathrm{e}^{2} n_{0}}{m_{\mathrm{e}}}$ and $n_{0}$ is the density of background plasma. Their group velocities, like those of Langmuir waves, are very small compared to the light speed $c$. It is extremely difficult for the oscillations to escape the plasma because the index of refraction for these waves is very nearly zero. Hence the Langmuir and the transverse modes are often grouped and called plasma oscillations. Thus, it is convenient to call the transverse modes of Eq. (1) transverse plasmons (EM wave). Due to very small group velocities, the dominant interactions between the transverse plasmons and Langmuir waves are strong; the interactions are connected with the scattering by

Send offprint requests to: $\mathrm{X}$. Q. $\mathrm{Li}$, e-mail: njxqli@jlonline.com electrons and ions $1+\mathrm{e} \rightleftharpoons \mathrm{p}+\mathrm{e}^{\prime}, 1+\mathrm{i} \rightleftharpoons \mathrm{p}+\mathrm{i}^{\prime}$ and the decay process $1+\mathrm{l}^{\prime} \rightleftharpoons \mathrm{p}$. Numerical calculations showed that there is a continuous transfer from Langmuir waves to the transverse plasmons and back and their energy densities are approximately the same, averaged over time, $W^{1} \approx W^{\mathrm{p}}$ (Kaplan \& Tsytovich 1973). In view of the physics, it is natural that the interactions lead to a tendency of energy equilibration over both of the Langmuir and the transverse plasmons with the same frequencies near $\omega_{\mathrm{pe}}$ and similar dispersion laws. On the other hand, for a plasma in thermal equilibrium, there is also a finite level of plasma waves, which represents degrees of freedom excited in thermal equilibrium. Langmuir plasmons are excited by the charged particles of the plasma through $\breve{C}$ erenkov processes $\mathrm{e} \rightarrow 1+\mathrm{e}^{\prime}$ (spontaneous emission) when they move due to their thermal energy and are then reabsorbed by the plasma due to Landau damping. A balance between the spontaneous emission and the induced absorption leads to a thermal level of Langmuir plasmons. The energy density of Langmuir plasmons in thermal equilibrium is (Kaplan \& Tsytovich 1973): $W_{\mathrm{T}}^{1}=\frac{n_{\mathrm{e}} k_{\mathrm{B}} T_{\mathrm{e}}}{6 \pi^{2} N_{\mathrm{D}}}$, where $n_{\mathrm{e}}$ the electron density, $T_{\mathrm{e}}$ the electron temperature, $N_{\mathrm{D}}$ the Debye number, and $k_{\mathrm{B}}$ the Boltzmann's constant. As a result, we may expect for a plasma on excited levels that

$\bar{W}^{\mathrm{p}} \equiv \frac{\left|\boldsymbol{E}^{\mathrm{p}}\right|^{2}}{8 \pi n_{\mathrm{e}} k_{\mathrm{B}} T_{\mathrm{e}}} \gg \frac{W_{\mathrm{T}}^{1}}{n_{\mathrm{e}} k_{\mathrm{B}} T_{\mathrm{e}}}=\frac{1}{6 \pi^{2} N_{\mathrm{D}}}$, 
or in the active region of the accretion disks,

$\bar{W}^{\mathrm{p}} \gg 3 \times 10^{-8}\left(\frac{n_{\mathrm{e}}}{10^{16}}\right)^{1 / 2}\left(\frac{T_{\mathrm{e}}}{3 \times 10^{7}}\right)^{-3 / 2}$,

where $\frac{\left|\boldsymbol{E}^{\mathrm{p}}\right|^{2}}{8 \pi}$ is the energy density of the transverse plasmons.

As we will show, the transverse plasma fields for a plasma on an exited level are modulationally unstable in the Lyapunov sense; the instabilities would develop and result eventually in self-similar collapse, giving rise to a more spatially intermittent magnetic flux. We will study this phenomena in both dynamic and kinetic cases, respectively, corresponding to the descriptions of different space scales and different astrophysical applications.

First we deal with the solar magnetic field, concerning ourself with magneto-hydrodynamics (MHD)theory which corresponds to the current precision of observations. It is shown that solar magnetic field is of a spatially intermittent nature. The intermittent flux fragments can be found all over the sun, showing that more than $90 \%$ of the flux occurs in strong-field form with strengths of 1-2 kG and sizes of 50-300 km in the photosphere (Stenflo 1989). The key to an unified understanding of the physics of solar atmosphere may be found in the MHD processes of the basic magnetic cells. It is likely that the flux is in a pre-concentrated state when it emerges from the solar interior to the surface due to the convective expulsion (Weiss 1966; Peckover \& Weiss 1972); hence a sort of instability would be responsible for the final concentration, from e.g. 0.1 to $1-2 \mathrm{kG}$ (Stenflo 1989). It is known that weak, vertical magnetic flux in the solar surface layers may be spontaneously concentrated into a $\mathrm{kG}$ state by the instability of convective collapse (Parker 1978; Spruit 1979). However, the convective instability or thermal instability is very difficult to explain that the magnetic pressure within the flux cells is much higher than the equipartition value-the convective turbulent pressure (Tagger et al. 1995). In their paper, Tagger et al. proposed an interesting instability mechanism for the filamentation of magnetic flux tubes in a weakly ionized plasma. Their result is somewhat phenomenological and qualitative, and it seems still necessary to investigate the process when non-linear effects of the magnetic field are considered.

It is well-established both from analytical and numerical works that magnetic fields in turbulent plasmas have a tendency toward self-organization in flux sheets or cells (Shramkowski \& Torkelsson 1996). Magnetic reconnection in the upper part of the emerged sections changes the topology from that of a connected "sea serpent" to that of a number of separated, smaller closed loops (Stenflo 1989). During the magnetic coalescence of multiple loops, the plasmas and magnetic flux may be compressed toward current sheets from both sides, driven by the Lorentz force, leading to a resistive instability. A reconnective annihilation of the magnetic field occurs and the magnetic energy is converted into the kinetic energy of particles, thermal energy of plasma and radiation by Ohmic dissipation, followed by forming many magnetic cells and enhanced plasmons including Langmuir and transverse ones in the thin current sheet. The cells and the strong turbulent plasmons can be intimately coupled by ponderomotive interaction. The equilibrium between the ponderomotive force and the Lorentz force will cause a instability for magnetic cells in the current sheet, which could create collapsing pattern and lead to the occurrence of $\mathrm{kG}$ state.

Another problem we will tackle in this paper is the magnetic viscosity and resistivity in accretion disks. It is generally accepted that astrophysical disks play a central role in active phenomena of young stellar objects (YSOs), cataclysmic variable stars, binary X-ray sources and active galactic nuclei (AGN). An anomalous viscosity is usually assigned to accretion disks, since normal microscopic viscosity are too small (by at least eight orders of magnitude) to sustain an astrophysically significant accretion flow. Furthermore, the magnetic diffusivity is also most probably anomalous (Lovelace et al. 1987). Current models usually make an ad hoc assumption, by introducing a parameter $\alpha_{\mathrm{t}}$ to parametrize our ignorance of the anomalous "turbulent viscosity" $\eta_{\mathrm{t}}$, as

$\eta_{\mathrm{t}}=\alpha_{\mathrm{t}} c_{\mathrm{s}} H \rho, \quad \alpha_{\mathrm{t}}=$ const.

where $H$ is the disk scale height, $c_{\mathrm{s}}$ the sound speed and $\rho$ the mass density. However ignorance makes stability analyses based on the $\alpha_{\mathrm{t}}$-formalism most problematic: what comprises the unperturbed state is impossible to define (Balbus \& Hawley 1998). The physical mechanisms (turbulence or more spatially intermittent magnetic fields) must be addressed. In particular we need to determine the functional dependence of $\alpha_{\mathrm{t}}$ on the physical variables of the disks.

As a candidate for the anomalous viscosity, the magnetic viscosity was first calculated from Keplerian fluid shear by Eardley \& Lightman (1975). Coroniti (1981) and Torkelsson (1993) extended the model by including magnetic buoyancy. These models concentrate on viscosity as a direct result of field line stretching by the Keplerian mean flow. But these investigations are still in the kinematical version and the relevant anomalous magnetic diffusivity was not obtained in these models. Lacking a pure hydrodynamic instability for turbulence, the Chandrasekhar-Balbus-Hawley magnetorotational instability (Balbus \& Hawley 1998) was proposed and might act as a turbulent transport mechanism in disks, with the advent of a weak magnetic field. However, it is not sensible to only dwell on magnetohydrodynamic studies. Investigations of microscopic and non-linear instabilities, especially of magnetic driving, resulting in anomalous viscosity, are also necessary. Thus we leave the discussion of magnetohydrodynamics and delve deeper into the plasma kinetic regime of accretion disks. We find that the non-linear collapse of the self-generated magnetic fields is responsible for generating the anomalous viscosity, and, as a bonus, an anomalous diffusivity. Furthermore, in our model, the functional dependence of $\alpha_{\mathrm{t}}$ on the physical variables of the disks is explicitly given if we still use $\alpha_{\mathrm{t}}$ to parametrize the viscosity. It is natural that one finds a physical mechanism that operates only on rather small scales of motion or of electric current, given the anomalous viscosity and resistivity of the matter with very different magnetic Prandtl numbers (Zahn 1991).

This paper is organized as follows. Section 2 is concerned with the collapsing instability for the solar magnetic cell in a current sheet; the basic ponderomotive effects on the evolution 
of the flux cell are illustrated in Sect. 2.1; and Sect. 2.2 discusses the collapse of the flux cell caused by transverse plasmons; we find the collapsed end state with field strengths of $\mathrm{kG}$ in Sect. 2.3. Section 3 deals with the collapsing instability of magnetic fields in accretion disks. The basic equations for selfgenerated fields are given in Sect. 3.1; the modulational instabilities caused by transverse pumping waves are explored in Sect. 3.2; in Sect. 3.3 we provide a self-similar solution for the collapse magnetic flux, and we determine the anomalous dc resistivity and viscosity from the self-generated spatially intermittent magnetic flux in Sect. 3.4. Section 4 gives the conclusions.

\section{Collapsing magnetic instability to solar intermittent flux}

\subsection{Ponderomotive effects}

For a weakly ionized plasma with electromagnetic oscillations, as in the photosphere, the basic equations are the fluid equations for a three-component plasma consisting of electrons, ions and neutral particles, supplemented by the Maxwell equations. Because of the large difference in electron and ion oscillation frequencies in the astrophysical plasma, the two timescale approximation is relevant for the charged particles. In this case all the field quantities, say, density, velocity, pressure, electric and magnetic fields, can be divided into fast and slow time-scale components, $A=\left(n_{\mathrm{e}}, n_{\mathrm{i}} ; \boldsymbol{v}_{\mathrm{e}}, \boldsymbol{v}_{\mathrm{i}} ; P_{\mathrm{e}}, P_{\mathrm{i}} ; \boldsymbol{E}, \boldsymbol{B}\right)=$ $A_{\mathrm{f}}+A_{\mathrm{s}}$, and it can be assumed that the ensemble average of the fast components over the slow time-scale vanishes: $\left\langle A_{\mathrm{f}}\right\rangle=0$. On the slow time-scale, a quasi-neutrality condition leads to $n_{\mathrm{s}}^{\mathrm{e}}=n_{\mathrm{s}}^{\mathrm{i}} \equiv n_{\mathrm{s}}$. Under these circumstances, estimating the relative magnitude of the terms in the fast- and slow-component equations, we can obtain the following transfer equation for the fast oscillation of electrons

$$
\begin{aligned}
\nabla \times \nabla \times v_{\mathrm{f}}^{\mathrm{e}}+\frac{1}{c^{2}} \frac{\partial^{2}}{\partial t^{2}} v_{\mathrm{f}}^{\mathrm{e}}= & -\frac{1}{c^{2}} \frac{4 \pi e^{2}}{m_{\mathrm{e}}} n_{\mathrm{s}} v_{\mathrm{f}}^{\mathrm{e}} \\
& +\frac{3 v_{T_{\mathrm{e}}}^{2}}{c^{2}} \frac{1}{n_{\mathrm{s}}} \nabla\left(\nabla \cdot\left(n_{\mathrm{s}} \boldsymbol{v}_{\mathrm{f}}^{\mathrm{e}}\right)\right)
\end{aligned}
$$

accompanied by the continuity and the momentum equations for the electrons and ions (Li \& Zhang 1997; Li et al. 1997):

$$
\begin{aligned}
\frac{\partial}{\partial t} n_{\mathrm{s}}+\nabla \cdot\left(n_{\mathrm{s}} \boldsymbol{v}_{\mathrm{s}}^{\mathrm{e}}\right)=0, & \frac{\partial}{\partial t} n_{\mathrm{s}}+\nabla \cdot\left(n_{\mathrm{s}} \mathbf{v}_{\mathrm{s}}^{\mathrm{i}}\right)=0, \\
\frac{D \boldsymbol{v}_{\mathrm{s}}^{\mathrm{e}}}{D t} \equiv & \frac{\partial}{\partial t} \boldsymbol{v}_{\mathrm{s}}^{\mathrm{e}}+\left(\boldsymbol{v}_{\mathrm{s}}^{\mathrm{e}} \cdot \nabla\right) \boldsymbol{v}_{\mathrm{s}}^{\mathrm{e}}=\frac{e}{m_{\mathrm{e}}}\left[\boldsymbol{E}_{\mathrm{s}}+\frac{1}{c} \boldsymbol{v}_{\mathrm{s}}^{\mathrm{e}} \times \boldsymbol{B}_{\mathrm{s}}\right] \\
& -\frac{\nabla P_{\mathrm{s}}^{\mathrm{e}}}{m_{\mathrm{e}} n_{\mathrm{s}}}+\boldsymbol{F}_{\mathrm{p}}^{\mathrm{e}}+\frac{1}{m_{\mathrm{e}} n_{\mathrm{s}}}\left(\boldsymbol{R}_{\mathrm{ei}}+\boldsymbol{R}_{\mathrm{en}}\right), \\
\frac{D \boldsymbol{v}_{\mathrm{s}}^{\mathrm{i}}}{D t} \equiv & \frac{\partial}{\partial t} \boldsymbol{v}_{\mathrm{s}}^{\mathrm{i}}+\left(\boldsymbol{v}_{\mathrm{s}}^{\mathrm{i}} \cdot \nabla\right) \boldsymbol{v}_{\mathrm{s}}^{\mathrm{i}}=-\frac{e}{m_{\mathrm{i}}}\left[\boldsymbol{E}_{\mathrm{s}}+\frac{1}{c} \boldsymbol{v}_{\mathrm{s}}^{\mathrm{i}} \times \boldsymbol{B}_{\mathrm{s}}\right] \\
& -\frac{\nabla P_{\mathrm{s}}^{\mathrm{i}}}{m_{\mathrm{i}} n_{\mathrm{s}}}+\frac{1}{m_{\mathrm{i}} n_{\mathrm{s}}}\left(-\boldsymbol{R}_{\mathrm{ei}}+\boldsymbol{R}_{\mathrm{in}}\right),
\end{aligned}
$$

where $\boldsymbol{R}_{\mathrm{ei}}$ and $\boldsymbol{R}_{\alpha \mathrm{n}}(\alpha=e, i)$ are the friction forces for electronions and charged-neutral particles, respectively; $v_{\mathrm{Te}}$ is the electron thermal velocity and $\boldsymbol{F}_{\mathrm{p}}^{\mathrm{e}}$ is the ponderomotive force describing the effect of the high frequency oscillations on the slow motion of the electron-fluid:

$F_{\mathrm{p}}^{\mathrm{e}}=-\frac{1}{2} \nabla\left\langle\left(v_{\mathrm{f}}^{\mathrm{e}}\right)^{2}\right\rangle$

Eliminating $\boldsymbol{E}_{\mathrm{s}}$ from Eqs. (8) and (9), one has

$$
\begin{aligned}
m_{\mathrm{e}} \frac{D \boldsymbol{v}_{\mathrm{s}}^{\mathrm{e}}}{D t}+m_{\mathrm{i}} \frac{D \boldsymbol{v}_{\mathrm{s}}^{\mathrm{i}}}{D t}= & \frac{\boldsymbol{j}_{\mathrm{s}} \times \boldsymbol{B}_{\mathrm{s}}}{c n_{0}}-\frac{1}{4} m_{\mathrm{e}} \nabla\left(\left|\boldsymbol{v}_{\mathrm{f} 0}\right|^{2}\right) \\
& -\frac{\nabla P_{\mathrm{s}}^{\mathrm{e}}+\nabla P_{\mathrm{s}}^{\mathrm{i}}}{n_{0}}+\frac{1}{n_{\mathrm{s}}} \boldsymbol{R}_{\mathrm{n}}
\end{aligned}
$$

here $\boldsymbol{j}_{\mathrm{s}}$ is the current density, $\nabla P_{\mathrm{s}}^{\alpha}=\gamma_{\alpha} T_{\alpha} \nabla n_{\mathrm{s}}^{\alpha}(\alpha=e, i)$, $\gamma_{\alpha}$ the specific heat ratio and $\boldsymbol{R}_{\mathrm{n}}=\left(\boldsymbol{R}_{\mathrm{en}}+\boldsymbol{R}_{\mathrm{in}}\right)$, with $\boldsymbol{R}_{\alpha \mathrm{n}}=$ $-m_{\alpha} n_{\mathrm{s}} v_{\alpha \mathrm{n}}\left(\boldsymbol{v}_{\alpha}-\boldsymbol{v}_{\mathrm{n}}\right)$, in which $v_{\alpha \mathrm{n}}$ is the collision frequency between charged particles and neutral ones. Expressing the fast oscillation velocity of the electron by

$\boldsymbol{v}_{\mathrm{f}}^{\mathrm{e}}=\frac{1}{2}\left[\boldsymbol{v}_{\mathrm{f} 0}(\boldsymbol{r}, t) \mathrm{e}^{i \omega_{0} t}+\right.$ c.c. $]$,

where $\omega_{0}$ is the fast oscillation frequency of the plasmons, $\omega_{0} \simeq \omega_{\text {pe }}$ and c.c. denotes complex conjunction of the first term, recalling that the equation of the lowest order on the fast time-scale, $\frac{\partial}{\partial t} \boldsymbol{v}_{\mathrm{f}}^{\mathrm{e}} \approx \frac{e}{m_{\mathrm{e}}} \boldsymbol{E}_{\mathrm{f}}$, and the Van der Pol types of complex electric vector in the waves, $\boldsymbol{E}_{\mathrm{f}}^{\mathrm{e}}=\frac{1}{2}\left[\boldsymbol{E}(\boldsymbol{r}, t) \mathrm{e}^{i \omega_{0} t}+\right.$ c.c. $]$, yield

$\left\langle\left(\boldsymbol{v}_{\mathrm{f}}^{\mathrm{e}}\right)^{2}\right\rangle=\frac{1}{2}\left|\boldsymbol{v}_{\mathrm{f} 0}\right|^{2}, \quad \boldsymbol{v}_{\mathrm{f} 0} \simeq \frac{-i e}{m_{\mathrm{e}} \omega_{0}} \boldsymbol{E}$.

In this case Eq. (5) becomes

$$
\begin{aligned}
2 i \omega_{\mathrm{pe}} \frac{\partial}{\partial t} \boldsymbol{v}_{\mathrm{f} 0}+ & c^{2} \nabla \times \nabla \times \boldsymbol{v}_{\mathrm{f} 0} \\
& -3 v_{T_{\mathrm{e}}}^{2} \nabla\left(\nabla \cdot \boldsymbol{v}_{\mathrm{f} 0}\right)+\frac{\delta n}{n_{0}} \omega_{\mathrm{pe}} \boldsymbol{v}_{\mathrm{f} 0}=0
\end{aligned}
$$

with $\left|\frac{1}{\omega_{0}} \frac{\partial}{\partial t} \ln v_{\mathrm{fo}}\right| \ll 1$, where $\delta n=n_{\mathrm{s}}-n_{0}$, which is the slow disturbance of density in the waves.

For the purpose of this study, we have assumed that the turbulence parameter $\bar{W}$, which expresses the excitation of the plasmons as a fraction of the total thermal energy of the plasma, satisfies

$$
\begin{aligned}
10^{-3}\left(\frac{n_{\mathrm{e}}}{10^{14} \mathrm{~cm}^{-3}}\right)^{1 / 2}\left(\frac{T_{\mathrm{e}}}{6520 \mathrm{~K}}\right)^{-3 / 2} & \\
=\frac{W_{\mathrm{T}}^{1}}{n_{\mathrm{e}} k_{\mathrm{B}} T_{\mathrm{e}}} & \ll \bar{W}=\frac{|\boldsymbol{E}|^{2}}{4 \pi n_{\mathrm{s}} T_{\mathrm{e}}} \approx \frac{\left|\boldsymbol{v}_{\mathrm{f} 0}\right|^{2}}{v_{\mathrm{Te}}^{2}}<1,
\end{aligned}
$$

and the electron plasma frequency $\omega_{\mathrm{pe}}$ is much greater than the cyclotron frequency $\omega_{\mathrm{Be}}, \omega_{\mathrm{pe}} \gg \omega_{\mathrm{Be}}$. Since the collision terms associated with the neutral particles are related to their motions, the continuity and the momentum equations for the neutral particles are:

$$
\frac{\partial n_{\mathrm{n}}}{\partial t}+\nabla \cdot\left(n_{\mathrm{n}} \boldsymbol{v}_{\mathrm{n}}\right)=0
$$

$m_{\mathrm{n}} n_{\mathrm{n}}\left[\frac{\partial}{\partial t} \boldsymbol{v}_{\mathrm{n}}+\left(\boldsymbol{v}_{\mathrm{n}} \cdot \nabla\right) \boldsymbol{v}_{\mathrm{n}}\right]=-\nabla P_{\mathrm{n}}-\boldsymbol{R}_{\mathrm{n}}$ 
To complete the Eq. (14) we need a further expression of the nonlinear term $\propto \frac{\delta n}{n_{0}} \boldsymbol{v}_{\mathrm{f} 0}$. In the case of a thin current sheet with flow and plasmons, linearizing with respect to $v, D / D t \approx \partial / \partial t$, multiplying by $\mathrm{d} z$ and integrating from $z=-z_{0}$ to $z=z_{0}$, from Eq. (11) we obtain

$$
\int_{-z_{0}}^{z_{0}} \frac{\partial}{\partial z}\left(\frac{1}{4} m_{\mathrm{e}}\left|\boldsymbol{v}_{\mathrm{f} 0}\right|^{2}+\frac{\delta n}{n_{0}} m_{\mathrm{i}} c_{\mathrm{s}}^{2}\right) \mathrm{d} z=0
$$

with $c_{\mathrm{s}}^{2}=\left(\gamma_{\mathrm{e}} k_{\mathrm{B}} T_{\mathrm{e}}+\gamma_{\mathrm{i}} k_{\mathrm{B}} T_{\mathrm{i}}\right) / m_{\mathrm{i}}$, where we have neglected the following terms due to $z_{0} \rightarrow 0: \int_{-z_{0}}^{z_{0}} \boldsymbol{v}_{\mathrm{s}} \mathrm{d} z=2 z_{0}<\boldsymbol{v}_{\mathrm{s}}>_{z} \rightarrow 0$, $\int_{-z_{0}}^{z_{0}}\left(\boldsymbol{j}_{\mathrm{s}} \times \boldsymbol{B}_{\mathrm{s}}\right) \mathrm{d} z=2 z_{0}<\boldsymbol{j}_{\mathrm{s}} \times \boldsymbol{B}_{\mathrm{s}}>_{z} \rightarrow 0, \int_{-z_{0}}^{z_{0}} \boldsymbol{R}_{n} \mathrm{~d} z \propto 2 z_{0}<$ $\boldsymbol{v}_{\alpha}-\boldsymbol{v}_{\mathrm{n}}>_{z} \rightarrow 0$. Then we find

$$
\frac{\delta n(x, y, z)}{n_{0}}=-\frac{m_{\mathrm{e}} / m_{\mathrm{i}}}{4 c_{\mathrm{s}}^{2}}\left|\boldsymbol{v}_{\mathrm{f} 0}(x, y, z)\right|^{2}
$$

In fact, this result was also found by Sotnikov \& Krasnoselskikh for the static limit (see Shapiro \& Shevchenko 1984). By using Eq. (18) we can write Eq. (14) in the form

$$
\begin{aligned}
i \frac{\partial}{\partial \tau} \boldsymbol{v}_{\mathrm{f} 0}^{\prime}+ & \alpha_{\mathrm{Te}} \nabla^{\prime} \times \nabla^{\prime} \times \boldsymbol{v}_{\mathrm{f} 0}^{\prime} \\
& -\nabla^{\prime}\left(\nabla^{\prime} \cdot \boldsymbol{v}_{\mathrm{f} 0}^{\prime}\right)-\left|\boldsymbol{v}_{\mathrm{f} 0}^{\prime}\right|^{2} \boldsymbol{v}_{\mathrm{f} 0}^{\prime}=0
\end{aligned}
$$

with

$\boldsymbol{r}^{\prime}=\frac{2}{3} \sqrt{\mu} k_{\mathrm{d}} \boldsymbol{r}, \tau=\frac{2}{3} \mu \omega_{\mathrm{pe}} t, \alpha_{\mathrm{Te}}=\frac{c^{2}}{3 v_{\mathrm{Te}}^{2}}$,

$\mu=\frac{m_{\mathrm{e}}}{m_{\mathrm{i}}}, \boldsymbol{v}_{\mathrm{f} 0}^{\prime}=\frac{\sqrt{3} \boldsymbol{v}_{\mathrm{f} 0}}{4 \sqrt{\mu} v_{\mathrm{Te}}}$,

where $k_{\mathrm{d}}$ is the Debye wave number.

For this three-element mixed fluid composed of electrons, ions and neutral particles, we can introduce a velocity related to the center of mass:

$$
\boldsymbol{U}=\frac{\rho_{\mathrm{e}} \boldsymbol{v}_{\mathrm{e}}+\rho_{\mathrm{i}} \boldsymbol{v}_{\mathrm{i}}+\rho_{\mathrm{n}} \boldsymbol{v}_{\mathrm{n}}}{\rho},
$$

where $\rho$ is the total mass density $\rho=\rho_{\mathrm{e}}+\rho_{\mathrm{i}}+\rho_{\mathrm{n}} \equiv n_{\mathrm{e}} m_{\mathrm{e}}+$ $n_{\mathrm{i}} m_{\mathrm{i}}+n_{\mathrm{n}} m_{\mathrm{n}}$. By a standard procedure (Li \& Wu 1989) we may obtain a set of equations for a global coupling MHD with ponderomotive force by combining Eqs. (6-9), (16) and (17). The relevant equations are ( $\mathrm{Li}$ et al. 1997):

$$
\begin{aligned}
\frac{\partial}{\partial t} \rho+\nabla \cdot & (\rho \boldsymbol{U})=0 \\
\rho\left[\frac{\partial}{\partial t} \boldsymbol{U}+\right. & (\boldsymbol{U} \cdot \nabla) \boldsymbol{U}]=\frac{1}{4 \pi}(\nabla \times \boldsymbol{B}) \\
& \times \boldsymbol{B}-\nabla P-\frac{1}{4} \frac{m_{\mathrm{e}}}{m_{\mathrm{i}}} \rho_{\mathrm{ig}} \nabla\left(\left|\boldsymbol{v}_{\mathrm{f} 0}\right|^{2}\right),
\end{aligned}
$$

where $\rho_{\mathrm{ig}}\left(\approx \rho_{\mathrm{i}}\right)$ is the mass density of the electro-conductive fluid composed of electrons and ions; $\boldsymbol{B}=\boldsymbol{B}_{\mathrm{s}}$, and $P$ is the total pressure related to the mass-center system.

\subsection{Magnetic flux cell collapse}

The field structure within a magnetic element is circular, as assumed by Coroniti (1981), which is described by the simple vector potential

$$
A_{z}=\frac{B_{0}(t)}{2 \ell}\left(x^{2}+y^{2}\right), \quad\left(x^{2}+y^{2} \leq \ell^{2}\right),
$$

where $B_{0}(t)$ is the characteristic magnetic field strength, which, in general, is dependent on time $t ; \ell(\gg \delta)$ is the length scale of the flux cell in the $(x, y)$ plane and $\delta$ is the vertical extent. The corresponding magnetic field components are

$B_{x}=-B_{0}(t) \frac{y}{\ell}, B_{y}=B_{0}(t) \frac{x}{\ell}$,

which is not a force-free one. Usually one can assume that the initial magnetic pressure is much less than the total plasma thermal pressure $P$ in the photosphere

$\widetilde{\beta} \equiv \frac{P}{B^{2}(t) / 8 \pi}>1$

Thus, the flux cell may interact with the plasmons, and this causes an equilibrium. Under the condition (25), we have from Eq. (22)

$\frac{1}{4 \pi}(\nabla \times \boldsymbol{B}) \times \boldsymbol{B}=\frac{1}{4} \mu \rho_{\mathrm{i}} \nabla\left(\left|\boldsymbol{v}_{\mathrm{f} 0}\right|^{2}\right)$.

We will later demonstrate that this equilibrium is indeed satisfied.

As we have mentioned, many different types of wave may grow in a reconnecting magnetic field and produce a turbulent environment within the thin current sheet. We may assume that there are plasmons oscillating along the $z$-direction in the sheet: $\boldsymbol{v}_{\mathrm{f} 0}^{\prime}=v_{\mathrm{f} z} z$ and $v_{\mathrm{f} z} \propto \operatorname{sech}\left(z \sigma_{0}\right)$ with $\sigma_{0} \gg 1$ (see (30) and (31) below). Thus, the divergence of the fast oscillation velocity, $\nabla \cdot \boldsymbol{v}_{\mathrm{f} 0}^{\prime} \propto-\sec h\left(z \sigma_{0}\right) \tanh \left(z \sigma_{0}\right)$, vanishes near the surfaces of the sheet. Under the circumstance of transverse plasmons, Eq. (19) becomes

$i \frac{\partial}{\partial \tau} v_{\mathrm{f} z}+\frac{1}{2} \nabla^{2}\left(v_{\mathrm{f} z}\right)+\left|v_{\mathrm{f} z}\right|^{2} v_{\mathrm{f} z}=0$,

where we have taken the complex conjunction and omitted the conjunction asterisk " $*$ " and the symbols " ' ", with $\nabla^{2}=\frac{\partial^{2}}{\partial \xi_{x}^{2}}+\frac{\partial^{2}}{\partial \xi_{y}^{2}}+\frac{\partial^{2}}{\partial \xi_{z}^{2}}$ and $\boldsymbol{\xi}=\boldsymbol{r}^{\prime} / \sqrt{2 \alpha_{\mathrm{Te}}}$. In the framework of Eq. (19) the transverse plasmon field is unstable in the Lyapunov sense to a finite-amplitude, monochromatic pumping wave $\boldsymbol{v}_{\mathrm{f} 0}^{0}\left(\right.$ or $\boldsymbol{E}_{0}^{\mathrm{p}}$, see (13)). It can be seen (Li 1989) that the modulational instability occurs when and only when $k^{2}<2\left|\boldsymbol{v}_{\mathrm{f} 0}^{0}\right|^{2}$,or, in dimensional units,

$\frac{\left|\boldsymbol{v}_{\mathrm{f} 0}^{0}\right|^{2}}{v_{\mathrm{Te}}^{2}}>6\left(\frac{k}{k_{\mathrm{d}}}\right)^{2}$

and the maximum growth rate and corresponding wave number of the instability for the longitudinal perturbation $\left(\boldsymbol{k} \| \boldsymbol{v}_{\mathrm{f} 0}^{0}\right)$ are

$$
\frac{\gamma_{\mathrm{max}}}{\omega_{\mathrm{pe}}}=\frac{1}{8} \frac{\left|\boldsymbol{v}_{\mathrm{f} 0}^{0}\right|^{2}}{v_{\mathrm{Te}}^{2}}, \quad \frac{k_{\mathrm{max}}^{1}}{k_{\mathrm{d}}}=\frac{1}{2 \sqrt{3}} \sqrt{\frac{\left|\boldsymbol{v}_{\mathrm{f} 0}^{0}\right|^{2}}{v_{\mathrm{Te}}^{2}}} .
$$


One can see from condition (28) that this instability is the zerothreshold instability for the finite-amplitude, monochromatic wave. The modulation of the perturbed pumping field, occurring as a result of instability, leads to the formation of field localization. And the nonlinear development of the modulational instability, for the two or three dimensional case, leads to collapse (Li et al. 1995). This causes an implosion of the waves and gives rise to spatially intermittent field structures with various intensities, very similar to chaotic or turbulent patterns. The numerical simulations illustrate this complex turbulence pattern .

A self-similar solution of the non-linear Schrodinger Eq. (27) was obtained first by Gorev et al. (1976). Now we will give an extension of their solution for our purpose. The solution is written in the following form:

$v_{\mathrm{f} z}=\sqrt{\sigma(R, z, \tau)} \exp [i S(R, \tau)]$

$\sigma=\sigma_{0}^{2}(R, \tau) \sec h^{2}\left(z \sigma_{0}\right), \quad R^{2}=\xi_{x}^{2}+\xi_{y}^{2}, \quad z=\xi_{z}$,

where the amplitude $\sigma_{0}(R, \tau)$ is a slowly varying function with respect to the phase function $S(R, \tau)$, i.e.,

$\left|\frac{\partial S}{\partial R}\right| \gg\left|\frac{\partial \sigma}{\partial R}\right| \cdot$

Making an ansatz for self-similar collapse (Gorev et al. 1976):

$\sigma_{0}=\left(\tau_{0}-\tau\right)^{-2 / 3} V(\zeta), \quad S=\left(\tau_{0}-\tau\right)^{-1 / 3} \psi(\zeta)$,

with

$\zeta=\frac{R}{\left(\tau_{0}-\tau\right)^{1 / 3}}$,

and substituting it into Eq. (27), after integrating the vertical scale, we obtain

$\psi+\zeta \frac{\mathrm{d} \psi}{\mathrm{d} \zeta}+\frac{1}{2}\left(\frac{\mathrm{d} \psi}{\mathrm{d} \zeta}\right)^{2}=\frac{3}{2} V^{2}$

$\frac{2}{3}+\frac{\zeta}{3} \frac{1}{V} \frac{\mathrm{d} V}{\mathrm{~d} \zeta}+\frac{1}{\zeta} \frac{\mathrm{d} \psi}{\mathrm{d} \zeta}+\frac{\mathrm{d}^{2} \psi}{\mathrm{d} \zeta^{2}}=0$

Putting $\psi=a+b \zeta^{2}$ and $\frac{3 b(1+2 b)}{a}=-\varepsilon^{2}$, and dropping the term $\frac{\zeta}{3} \frac{1}{V} \frac{\mathrm{d} V}{\mathrm{~d} \zeta} \sim \frac{\varepsilon^{2} \zeta^{2}}{1-\varepsilon^{2} \zeta^{2}}$ if $\varepsilon^{2} \zeta^{2} \ll 1$, yields $a \simeq \frac{1}{3 \varepsilon^{2}}, b \simeq-1 / 6$. As a result we find

$\sigma_{0}=\frac{\sqrt{2}}{3 \varepsilon}\left(\tau_{0}-\tau\right)^{-2 / 3}\left[1-\varepsilon^{2} \frac{R^{2}}{\left(\tau_{0}-\tau\right)^{2 / 3}}\right]^{1 / 2}$,

$S=\frac{1}{3 \varepsilon^{2}}\left(\tau_{0}-\tau\right)^{-1 / 3}\left[1-\frac{\varepsilon^{2}}{2} \frac{R^{2}}{\left(\tau_{0}-\tau\right)^{2 / 3}}\right]$.

The case with $\varepsilon=1$ was obtained by Gorev et al. (1976) which is valid only for $\zeta<1$. Now the extended solution above is also available for $\zeta>1$, provided $\varepsilon^{2} \zeta^{2} \ll 1$. Thus, at the plane of the sheet

$\left|\boldsymbol{v}_{f z}\right|^{2}=\sigma_{0}^{2}=\frac{2}{9 \varepsilon^{2}}\left(\tau_{0}-\tau\right)^{-4 / 3}\left[1-\varepsilon^{2} \frac{R^{2}}{\left(\tau_{0}-\tau\right)^{2 / 3}}\right]$ or, in dimensional units,

$$
\begin{aligned}
\left|\boldsymbol{v}_{\mathrm{f} z}\right|^{2} / v_{\mathrm{Te}}^{2}= & \frac{16}{9}\left(\frac{3}{2}\right)^{1 / 3} \frac{\mu^{-1 / 3}}{\varepsilon^{2}}\left(\widetilde{\tau_{0}}-\widetilde{\tau}\right)^{-4 / 3} \\
& \times\left[1-\left(\frac{2}{81}\right)^{1 / 3} \mu^{1 / 3} \varepsilon^{2} \frac{3 k_{0}^{2}\left(x^{2}+y^{2}\right)}{\left(\widetilde{\tau_{0}}-\widetilde{\tau}\right)^{2 / 3}}\right]
\end{aligned}
$$

with $\widetilde{\tau}=\omega_{\text {pe }} t$ and $k_{0}=\omega_{\text {pe }} / c$. One sees from (24), (26) and (37) that there indeed is an equilibrium between ponderomotive force and the Lorentz force within the sheet. In this case one has

$$
\begin{aligned}
\bar{B}^{2} / 8 \pi & =2\left(\bar{B}_{y}^{2} / 8 \pi\right)=\frac{1}{4 \pi}\left[\frac{1}{2 \ell} \int_{-\ell}^{\ell} B_{y} \mathrm{~d} x\right]^{2} \\
& =\frac{1}{16 \pi} B_{0}^{2}(t)=\frac{1}{18}\left(\frac{k_{0} \ell}{\widetilde{\tau}_{0}}\right)^{2} \frac{P_{\mathrm{i}}}{\left(1-t / t_{0}\right)^{2}}
\end{aligned}
$$

with $P_{\mathrm{i}}=2 n_{0} k_{\mathrm{B}} T$. Taking

$\widetilde{\tau}_{0}=\frac{k_{0} \ell}{3 \sqrt{2}}$

yields from (38) for the initial state $(t=0)$

$\bar{B}^{2}(0) / 8 \pi=P_{\mathrm{i}}$,

which corresponds to the magnetic field strength of $70 \mathrm{G}$ in the photosphere.

\subsection{KG flux fragments with small scales}

The collapse results from the development of the modulational instabilities. Hence we may identify the $\tau_{\text {coll }} \equiv\left(\widetilde{\tau_{0}}-\widetilde{\tau}\right)$ as the scale $\left(\widetilde{\tau_{0}}-\widetilde{\tau}\right)_{\min }$ to stop collapse, which corresponds to $\bar{W}^{\mathrm{p}} \sim 1$. On the one hand, the derivation of our basic Eqs. (19) and (22) breaks down as soon as $\bar{W}^{\mathrm{p}}$ is no longer small as compared to unity. On the other hand, the parameter $\bar{W}^{\mathrm{p}}$ would be limited by unity: the strong field with $\bar{W}^{\mathrm{p}}$ in excess thereof would make the nonlinear interactions very strong and the time-scale of interactions very short; physically an energy flow arises, which would lead to an increment of thermal energy $n_{\mathrm{e}} T_{\mathrm{e}}$ in a very short period, and then $\bar{W}^{\mathrm{p}}$ quickly drops to below unity again (Tsytovich 1977). Therefore the minimum time-scale of collapse motion could be got from the self-similar solution (37) as

$\left(1-t / t_{0}\right)_{\min }^{2} \simeq\left(\frac{16}{9}\right)^{3 / 2}\left(\frac{3}{2}\right)^{1 / 2} \mu^{-1 / 2} \frac{\varepsilon^{-3}}{\widetilde{\tau}_{0}^{2}}$.

At the same time, the magnetic field gets stronger during the collapsing process and the parameter $\widetilde{\beta}$ decreases. Then, one sees from (25) that the equilibrium (26) is no longer valid when $\widetilde{\beta} \sim 1$. Hence we can get another limit for the minimum timescale from (38)

$\left(1-t / t_{0}\right)_{\min }^{2} \simeq P_{\mathrm{i}} / P$

with

$\bar{B}_{\max }^{2} / 8 \pi=P$.

Therefore, the collapsed end state (43) in the photosphere has a field strength of 1-2 kG. Now we have seen that when the flux 
cell of $70 \mathrm{G}$ in the preconcentrated state emerges from the solar interior to the surface it undergoes collapse, until the end state for which total gas pressure in the photosphere is equal to the magnetic pressure of the field of strength of $1-2 \mathrm{kG}$.

The form of solution in (33) is called "self-similar" because the initial spatial shape $V\left(\frac{R}{\tau_{0}^{1 / 3}}\right)$ is preserved at later times, although contracted, since $\tau_{0}{ }^{1 / 3}$ is replaced by the smaller scale $\left(\tau_{0}-\tau\right)^{1 / 3}$. The solution $\sigma_{0}$ is therefore similar to itself at later times, except it becomes narrower, and also larger, because of the factor $\left(\tau_{0}-\tau\right)^{-2 / 3}$ in front of $V$. The scaling condition, $\varepsilon \zeta<1$, of self-similar collapse for enhanced transverse plasmons in dimensional units becomes

$\varepsilon \zeta=\varepsilon\left[\left(\frac{2}{3} \mu\right)^{1 / 6} \frac{k_{0} r}{\left(\widetilde{\tau_{0}}-\widetilde{\tau}\right)^{1 / 3}}\right]<1$.

At the initial state with length scale $r_{0}$, in view of (41) and (42) one has

$r_{0}<\ell\left[4 \sqrt{2}\left(\frac{n_{\mathrm{H}}}{2 n_{0}}\right)^{1 / 3}\right]^{-1}$

in addition, in the case of similar collapsing of the plasmons for which $\zeta(0)=\zeta(t)$, the contracted spatial length scale is

$r_{\min }=r_{0}\left(1-t / t_{0}\right)_{\min }^{1 / 3}=\left(\frac{2 n_{0}}{n_{\mathrm{H}}}\right)^{1 / 6}$,

where $P \simeq n_{\mathrm{H}} k_{\mathrm{B}} T$ and $n_{\mathrm{H}}$ is the neutral hydrogen density. For the photosphere, taking $n_{0}=10^{14} \mathrm{~cm}^{-3}, n_{\mathrm{H}}=10^{17} \mathrm{~cm}^{-3}$ and the horizontal scale-length $\ell \sim 10^{4} \mathrm{~km}$ yields that $r_{0} \sim 200 \mathrm{~km}$ and $r_{\min } \sim 80 \mathrm{~km}$. Under this circumstance, the $0.1 \mathrm{kG}$ flux cells, with larger scale $r \gg r_{0}$, emerging at the surface from the solar interior become shredded due to the equilibrium with collapsing plasmons, and the flux is thereby contracted rather quickly to small scale, of the order of $100 \mathrm{~km}$, with magnitude in the $\mathrm{kG}$ range.

\section{Collapsing magnetic instability to the anomalous viscosity in accretion disks}

\subsection{Equations of self-generated magnetic flux}

Now we are to show the action of the self-similar collapse of the self-generated magnetic field by transverse plasmons on the problem of anomalous viscosity in accretion disks. Binary and AGN disks are highly ionized (Stepinski 1992). In such a plasma, the electron-ion mean free path $\lambda_{\mathrm{mfp}}$ and the electron collision frequency $v_{\mathrm{e}}$ in AGN disks are

$$
\begin{aligned}
& \lambda_{\mathrm{mfp}}=10^{3}\left(\frac{n_{\mathrm{e}}}{10^{16}}\right)^{-1}\left(\frac{T_{\mathrm{e}}}{3 \times 10^{7}}\right)^{2}\left(\frac{\ln \Lambda}{20}\right)^{-1} \quad(\mathrm{~cm}) \\
& \frac{v_{\mathrm{e}}}{\omega_{\mathrm{pe}}}=5.4 \times 10^{-7}\left(\frac{n_{\mathrm{e}}}{10^{16}}\right)^{1 / 2}\left(\frac{T_{\mathrm{e}}}{3 \times 10^{7}}\right)^{-3 / 2}\left(\frac{\ln \Lambda}{20}\right),
\end{aligned}
$$

where $\ln \Lambda$ is the Coulomb logarithm, $\omega_{\text {pe }}$ is electron plasma frequency, $n_{\mathrm{e}}\left(\mathrm{cm}^{-3}\right)$ and $T_{\mathrm{e}}(\mathrm{K})$ are the local electric density and temperature, $10^{16}\left(\mathrm{~cm}^{-3}\right)$ and $3 \times 10^{7}(\mathrm{~K})$ are their typical values in the a lower density warm layer ("active region") of the disks (Zhang et al. 2000). At present we are to study the generation of localized magnetic flux, with the characteristic scale size $l_{\mathrm{c}}$ (see Eq. (78) below), which is small compared to $\lambda_{\mathrm{mfp}}$; in this case, indeed, we must generally attack the problem as one of kinetic theory. On the other hand, the electric current system on such small scales is free to respond to the local shear stresses of Keplerian differential rotation. So the classical plasma kinetics are an appropriate description. And the growth rate of modulation instabilities $\gamma_{M}$ (see Eq. (81) below) is much larger than the electron collision frequency $v_{\mathrm{e}}$, hence it is reasonable to omit the collision term in the kinetic equations. In addition, it is shown that the fluctuating magnetic field can be orders of magnitude stronger than the global magnetic field in disks (Schramkowski \& Torkelsson 1996). Therefore, it is relevant to use the Vlasov equations and Maxwell equations, with Maxwellan plasma and a vanishingly small background magnetic field, to depict the wave-wave and wave-particle interactions. In a plasma with a Maxwellian distribution, which is considered as the equilibrium unperturbed state, coupling of the transverse plasmons may produce low-frequency fields and nonlinear currents, leading to the creation of magnetic fields. Hence we need to consider both the interactions of waveparticle and wave-wave couplings. The fluctuations by such kinetic processes are notoriously non-thermal. A similar scenario for kinetic descriptions appeared in the works of Hakimov \& Tsytovich (1976), to derive the standard Zakharov equations, and of Bel'kov \& Tsytovich (1979). Here we focus on the transverse modes. The starting point are Vlasov's equations

$\frac{\partial f_{\alpha}}{\partial t}+\boldsymbol{v} \frac{\partial f_{\alpha}}{\partial \boldsymbol{r}}+\boldsymbol{F} \frac{\partial f_{\alpha}}{\partial \boldsymbol{p}}=0, \quad(\alpha=\mathrm{e}, \mathrm{i})$,

$\boldsymbol{F}=e_{\alpha}\left[\boldsymbol{E}+\frac{\boldsymbol{v}}{c} \times \boldsymbol{B}\right]$,

where $f_{\alpha}$ is the particle distribution function

$\int f_{\alpha} \frac{\mathrm{d} \boldsymbol{p}}{(2 \pi)^{3}}=n_{\alpha}$

and $n_{\alpha}$ is the number density of plasma particles and $\boldsymbol{F}$ is electromagnetic force. It is customary to decompose $f_{\alpha}$ and $\boldsymbol{F}$ into two parts:

$f_{\alpha}=f_{\alpha}^{\mathrm{R}}+f_{\alpha}^{\mathrm{T}}, \quad \boldsymbol{F}=\boldsymbol{F}^{\mathrm{R}}+\boldsymbol{F}^{\mathrm{T}}$,

$f_{\alpha}^{\mathrm{R}}\left(\boldsymbol{F}^{\mathrm{R}}\right)$ is unperturbed component and $f_{\alpha}^{\mathrm{T}}\left(\boldsymbol{F}^{\mathrm{T}}\right)$ is perturbed one. As $f_{\alpha}$ and $\boldsymbol{F}$ are closely coupled through Maxwell equations and the current density equation

$\boldsymbol{j}=\sum_{\alpha} \int e_{\alpha} \boldsymbol{v} f_{\alpha} \frac{\mathrm{d} \boldsymbol{p}}{(2 \pi)^{3}}$

and the perturbed fields for the transverse mode $\boldsymbol{E}^{\mathrm{T}}=\boldsymbol{E}^{\mathrm{p}}$ is assumed weak, so that the energy density excited is much smaller than thermal one of the plasma, i.e.,

$\bar{W}^{\mathrm{p}} \equiv \frac{\left|\boldsymbol{E}^{\mathrm{p}}\right|^{2}}{8 \pi n_{\mathrm{e}} k_{\mathrm{B}} T_{\mathrm{e}}}<1$,

we may expand $f_{\alpha}^{\mathrm{T}}$ in powers of the perturbed field $\boldsymbol{E}^{\mathrm{T}}$

$f_{\alpha}^{\mathrm{T}}=\sum_{\alpha} f_{\alpha}^{\mathrm{T}(a)}$, 
where the index $a$ indicates that $f^{\mathrm{T}(a)}$ is proportional to the $a$ th power of $E^{\mathrm{T}}$. Expanding $A=(\boldsymbol{F}, f)$ in a Fourier series

$A(\boldsymbol{r}, t)=\int A_{k} \mathrm{e}^{-i \omega t+i k r} \mathrm{~d} k, \quad\left(A_{k}=A_{\boldsymbol{k}, \omega} \quad \mathrm{d} k=\mathrm{d} \boldsymbol{k} \mathrm{d} \omega\right)$,

and taking Eqs. (51), (53) and the Maxwell equations into consideration, we can find the following field equation for the transverse mode from Eq. (48)

$\left(k^{2} c^{2}-\omega^{2} \varepsilon_{k}^{\mathrm{t}}\right) E_{k}^{\mathrm{T}}=4 \pi i \omega\left[\boldsymbol{e}_{\boldsymbol{k}}^{\mathrm{t} *} \cdot\left(\boldsymbol{j}_{k}^{(2)}+\boldsymbol{j}_{k}^{(3)}\right)\right]$,

where $\boldsymbol{j}_{k}^{(2)}$ and $\boldsymbol{j}_{k}^{(3)}$ are the nonlinear currents (to second and third order), $\boldsymbol{e}_{\boldsymbol{k}}^{\mathrm{t}}$ is the polarization vector for the transverse mode and $\varepsilon_{k}^{\mathrm{t}}$ denotes the transverse part of the dielectric tensor. For the low-frequency $\left(v_{T_{\mathrm{e}}} \gg \omega / k \gg v_{T_{\mathrm{i}}}\right)$,

$\varepsilon_{k}^{\mathrm{t}} \approx 1-\frac{\omega_{\mathrm{pe}}^{2}}{k^{2} v_{T_{\mathrm{e}}}^{2}}\left(1+\frac{k^{2} v_{\mathrm{s}}^{2}}{\omega^{2}}\right)$,

and for high frequency $\left(\omega^{\mathrm{p}} \simeq \omega_{\mathrm{pe}} \gg k v_{T_{\mathrm{e}}}\right), \varepsilon_{k}^{\mathrm{t}} \approx 1-\frac{\omega_{\mathrm{pe}}^{2}}{\omega^{2}}$. After the separation of high-frequency transverse field $\boldsymbol{E}_{k}^{\mathrm{T}( \pm)}$ with the positive or negative frequency and low-frequency fields $E_{k}^{\mathrm{T}} \equiv$ $E_{k}^{\mathrm{Ts}}$ (one recalls $\delta \boldsymbol{B}_{k}^{\mathrm{s}}=\frac{c}{\omega} \boldsymbol{k} \times \boldsymbol{E}_{k}^{\mathrm{Ts}}$ ), solving the Vlasov equations by the perturbation method, from the field Eq. (55) we obtain the following equations (Li \& Ma 1993, 1994):

$$
\begin{aligned}
\left(\frac{\partial^{2}}{\partial \tau^{2}}-\nabla^{2}\right) n(\boldsymbol{\xi}, \tau) & =\nabla^{2}|\boldsymbol{E}(\xi, \tau)|^{2}, \\
i \frac{\partial}{\partial \tau} \boldsymbol{E}(\xi, \tau)- & \alpha \nabla \times \nabla \times \boldsymbol{E}(\xi, \tau) \\
= & n(\xi, \tau) \boldsymbol{E}(\xi, \tau)+i \boldsymbol{E}(\xi, \tau) \times \boldsymbol{B}(\xi, \tau),
\end{aligned}
$$

$$
\begin{aligned}
\left(-\frac{\partial^{2}}{\partial \tau^{2}}+\right. & \nabla \times \nabla \times) \boldsymbol{B}(\xi, \tau) \\
& =i \frac{2}{3} \nabla \times \nabla \times \frac{\partial}{\partial \tau}\left[\boldsymbol{E}(\xi, \tau) \times \boldsymbol{E}^{*}(\xi, \tau)\right]
\end{aligned}
$$

with

$\xi=\frac{2}{3} \sqrt{\mu} \frac{1}{d_{\mathrm{e}}} r, \quad \tau=\frac{2}{3} \mu \omega_{\mathrm{pe}} t$,

$\alpha=\frac{c^{2}}{3 v_{T_{\mathrm{e}}}^{2}}, \quad \mu=\frac{m_{\mathrm{e}}}{m_{\mathrm{i}}}, \quad n=\frac{3}{4 \mu} \frac{n^{\prime}}{n_{\mathrm{e}}}$,

$\boldsymbol{E}(\xi, \tau)=\frac{\sqrt{3} E^{\mathrm{p}}(\boldsymbol{r}, t)}{4\left(\pi \mu n_{\mathrm{e}} k_{\mathrm{B}} T_{\mathrm{e}}\right)^{1 / 2}}$,

$\boldsymbol{B}(\xi, \tau)=\frac{3 e}{4 \mu m_{\mathrm{e}} c \omega_{\mathrm{pe}}} \delta \boldsymbol{B}^{s}(\boldsymbol{r}, t)$,

where $n^{\prime}(\boldsymbol{r}, t)$ is the perturbed density caused by this low-frequency field produced by the transverse fields $\left(\nabla \cdot \boldsymbol{E}^{\mathrm{p}}(\boldsymbol{r}, t)=\mathbf{0}\right)$ with positive and negative high-frequencies, $\delta \boldsymbol{B}^{\mathrm{s}}(\boldsymbol{r}, t)$ is self-generated magnetic field with low-frequencies, $n_{\mathrm{e}}$ the unperturbed (local) plasma density, $e$ the electric charge and $\boldsymbol{E}^{\mathrm{p}}(\boldsymbol{r}, t)$ is the envelope of the high-frequency transverse field $\boldsymbol{E}_{k}^{\mathrm{T}(+)}$,

$\boldsymbol{E}^{\mathrm{p}}(\boldsymbol{r}, t) \mathrm{e}^{-i \omega_{\mathrm{pe}} t}=\int \boldsymbol{E}_{k}^{\mathrm{T}(+)} \mathrm{e}^{-i \omega t+i \boldsymbol{k} \cdot \boldsymbol{r}} \mathrm{d} k$
The turbulence parameter is given by

$\bar{W}^{\mathrm{p}} \equiv \frac{\left|\boldsymbol{E}^{\mathrm{p}}\right|^{2}}{8 \pi n_{\mathrm{e}} k_{\mathrm{B}} T_{\mathrm{e}}}=\frac{2}{3} \mu|\boldsymbol{E}|^{2}$.

In the case of the static limit (to neglect the term $\frac{\partial^{2}}{\partial \tau^{2}}$ ) for the low-frequency magnetic field, Eq. (59) is reduced to

$\boldsymbol{B}=i \frac{2}{3} \frac{\partial}{\partial \tau}\left(\boldsymbol{E} \times \boldsymbol{E}^{*}\right)$.

\subsection{Modulational instabilities for non-static limit}

The self-generated magnetic fields are unstable in the Lyapunov sense to the pumping wave,

$\boldsymbol{E}_{\mathrm{I}}=\boldsymbol{E}_{0} \exp \left(i \boldsymbol{k}_{0} \xi-i \omega_{0} \tau\right), \quad\left(n_{I}=0, \boldsymbol{B}_{\mathrm{I}}=0\right)$

with $\omega_{0}=\alpha k_{0}^{2}$ (the transverse mode (1) in dimensional units) and $\boldsymbol{k}_{0} \cdot \boldsymbol{E}_{0}=0$. In fact, we study the following forms of plan waves of perturbations:

$n_{\mathrm{II}}=\frac{1}{2} n\left[\mathrm{e}^{i \boldsymbol{k} \cdot \xi-i \omega \tau}+\mathrm{e}^{-i k \cdot \xi+i \omega \tau}\right]$

$\boldsymbol{B}_{\mathrm{II}}=\frac{1}{2} \boldsymbol{B}\left[\mathrm{e}^{i \boldsymbol{k} \cdot \xi-i \omega \tau}+\mathrm{e}^{-i \boldsymbol{k} \cdot \xi+i \omega \tau}\right]$,

$\delta \boldsymbol{E}=\left[\boldsymbol{E} \mathrm{e}^{i \boldsymbol{k} \cdot \xi-i \omega \tau}+\boldsymbol{E}^{+} \mathrm{e}^{-i \boldsymbol{k} \cdot \xi+i \omega \tau}\right] \mathrm{e}^{i \boldsymbol{k}_{0} \cdot \xi-i \omega_{0} \tau}$,

where $\boldsymbol{E}, \boldsymbol{E}^{+}$are transverse perturbations:

$\boldsymbol{E}=E \boldsymbol{e}_{1,} \quad \boldsymbol{E}^{+}=E^{*} \boldsymbol{e}_{2}^{+}, \quad \boldsymbol{e}_{1} \perp \boldsymbol{k}_{+}, \quad \boldsymbol{e}_{2}^{+} \perp \boldsymbol{k}_{-}$,

with $\boldsymbol{k}_{ \pm}=\boldsymbol{k} \pm \boldsymbol{k}_{0}$, while $\boldsymbol{e}_{1}$ and $\boldsymbol{e}_{2}^{+}$are real unit vectors. Linearizing Eqs. (57-59) with respect to the perturbations we can obtain the following dispersion relation,

$\left[\omega^{2}-k^{2}-\frac{2 \alpha k^{4}\left|\boldsymbol{E}_{0}\right|^{2}}{\left(\omega-2 \alpha \mathbf{k} \cdot \mathbf{k}_{0}\right)^{2}-\alpha^{2} k^{4}}\right]\left[2\left(\omega+\alpha k^{2}-2 \alpha \boldsymbol{k} \cdot \boldsymbol{k}_{0}\right)\left(\boldsymbol{e}_{0} \cdot \boldsymbol{e}_{2}^{+}\right)\right.$

$\left[-i \frac{4}{3} \frac{1}{\left(1+\omega^{2} / k^{2}\right)} \omega\left|\boldsymbol{E}_{0}\right|^{2} G(\theta)\right]=i\left|\boldsymbol{E}_{0}\right|^{4} \frac{\frac{8}{3} \alpha k^{4} \omega G(\theta)}{\left[\left(\omega-2 \alpha k \cdot k_{0}\right)^{2}-\alpha^{2} k^{2}\right]} \frac{1}{\left(1+\omega^{2} / k^{2}\right)}$ with

$G(\theta)=\left[\left(\boldsymbol{e}_{0}^{*} \cdot \boldsymbol{e}_{2}^{+}\right)\left(\boldsymbol{e}_{0} \cdot \boldsymbol{e}_{0}\right)-\left(\boldsymbol{e}_{0} \cdot \boldsymbol{e}_{2}^{+}\right)\right]+\frac{E_{0}^{*}}{E_{0}}\left[\left(\boldsymbol{e}_{0} \cdot \boldsymbol{e}_{1}\right)\left(\boldsymbol{e}_{0}^{*} \cdot \boldsymbol{e}_{0}^{*}\right)-\right.$ $\left.\left(\boldsymbol{e}_{0}^{*} \cdot \boldsymbol{e}_{1}\right)\right], \quad \boldsymbol{e}_{0}=\frac{\boldsymbol{E}_{0}}{E_{0}}, \quad \boldsymbol{e}_{0}^{*}=\frac{\boldsymbol{E}_{0}^{*}}{E_{0}^{*}}$.

The dispersion equation is quite complicated in general. Let's for the moment restrict ourselves to an important special case of $\boldsymbol{k} \perp \boldsymbol{k}_{0},|\boldsymbol{k}| \gg\left|\boldsymbol{k}_{0}\right|$ and $\boldsymbol{e}_{0}=\frac{\boldsymbol{E}_{0}}{E_{0}}$ is real as well as $\boldsymbol{e}_{0} \| \boldsymbol{k}$; in this case the dispersion relation is reduced to

$\omega^{4}-\left(\alpha^{2} k^{4}+k^{2}\right) \omega^{2}-2 \alpha k^{4}\left|\boldsymbol{E}_{0}\right|^{2}+\alpha^{2} k^{6}=0$.

Thus, an instability occurs if and only if

$\left|\boldsymbol{E}_{0}\right|^{2}>\frac{\alpha}{2} k^{2}$.

We find growth rate $\gamma(\equiv \operatorname{Im} \omega)$ to be

$2 \gamma^{2}=\left[\left(\alpha^{2} k^{2}-1\right)^{2} k^{4}+8 \alpha\left|\boldsymbol{E}_{0}\right|^{2} k^{4}\right]^{\frac{1}{2}}-\left(\alpha^{2} k^{2}+1\right) k^{2}$.

The condition under which the growth rate $\gamma^{2}$ is a maximum is

$x^{3}-2 x^{2}+x(10 \beta+1)-\beta(8 \beta+1)=0$,

with $x \equiv \alpha^{2} k^{2}, \quad \beta \equiv \alpha\left|\boldsymbol{E}_{0}\right|^{2}$. When $\beta \gg 1$, which corresponds to a strong turbulence level, we can neglect the $x^{2}$-term and the $x$-term in Eq. (73) and find

$k_{\max } \approx \sqrt{2} \alpha^{-\frac{2}{3}}\left(\left|\boldsymbol{E}_{0}\right|^{2}\right)^{\frac{1}{3}} ;$ 
then it yields from (72)

$\gamma_{\max } \approx \sqrt{2} \alpha^{-\frac{1}{2}}\left(\left|\boldsymbol{E}_{0}\right|^{2}\right)^{\frac{1}{2}}$.

This instability is modulational for $k_{0} \ll k$; and one has

$\gamma_{\max } / k_{\max }=\beta^{1 / 6}>1$,

corresponding to the non-static limit. We attain to that the modulational instabilities occur if and only if (see (71))

$\xi>\xi_{\text {crit }} \equiv 2 \pi \frac{\sqrt{\alpha}}{\sqrt{2\left|\boldsymbol{E}_{0}\right|^{2}}}$,

and $\xi_{\text {crit }}$ is a critical scale size the fields building up to, at which the instabilities are suppressed; or in dimensional units,

$$
\begin{aligned}
l>l_{\mathrm{c}} & \equiv \sqrt{3} \pi d_{\mathrm{e}} \sqrt{\alpha}\left(\bar{W}_{0}^{\mathrm{p}}\right)^{-1 / 2} \\
& =5.4 \cdot\left(\frac{n_{\mathrm{e}}}{10^{16}}\right)^{-1 / 2}\left(\frac{\bar{W}_{0}^{\mathrm{p}}}{10^{-5}}\right)^{-1 / 2} \cdot(\mathrm{cm}) .
\end{aligned}
$$

And then in the case of non-static limit the maximum growth rate $\gamma_{\max }$, and the scale size $\xi_{\mathrm{M}}$, at which the maximum growth rate occurs, are (see (75))

$\gamma_{\mathrm{M}} \equiv \gamma_{\max }=\frac{\sqrt{2\left|\boldsymbol{E}_{0}\right|^{2}}}{\sqrt{\alpha}}$

$\xi_{\mathrm{M}} \equiv \frac{2 \pi}{k_{\max }}=\frac{2 \pi}{\sqrt{2}\left(\left|\boldsymbol{E}_{0}\right|^{2}\right)^{1 / 3} \alpha^{-2 / 3}}$,

under the condition

$\beta \equiv \alpha\left|\boldsymbol{E}_{0}\right|^{2} \gg 1$

Or, in dimensional units (by use of (60))

$$
\begin{aligned}
\frac{\gamma_{\mathrm{M}}}{\omega_{\mathrm{pe}}} & =2 \mu^{1 / 2} \frac{v_{\mathrm{T}_{\mathrm{e}}}}{c} \sqrt{\bar{W}_{0}^{\mathrm{p}}} \\
& =1.83 \times 10^{-5}\left(\frac{T_{\mathrm{e}}}{3 \times 10^{7}}\right)^{1 / 2}\left(\frac{\bar{W}_{0}^{\mathrm{p}}}{10^{-5}}\right)^{1 / 2}
\end{aligned}
$$

with

$$
\bar{W}_{0}^{\mathrm{p}}=\frac{\left|\boldsymbol{E}_{0}^{\mathrm{p}}\right|^{2}}{8 \pi n_{\mathrm{e}} k_{\mathrm{B}} T_{\mathrm{e}}} \gg 2 \mu \frac{v_{T_{\mathrm{e}}}^{2}}{c^{2}}=5 \times 10^{-6}\left(\frac{T_{\mathrm{e}}}{3 \times 10^{7}}\right) \text {. }
$$

For most astrophysical disks, say AGN disks, the condition (82), i.e. (80), could be satisfied indeed, as seen from (3).

\subsection{Self-similar collapse}

Now let us find a self-similar solution of Eqs. (57)-(59). It is analytically proved that the self-generated magnetic fields show self-collapse behavior (Li \& Ma 1994). Numerical simulations (Li \& Ma 1993, 1994) illustrate clearly the collapse behavior and the evolution of the solution of Eqs. (57), (58) and (65) for a given initial condition, showing that the nonlinear collapse gives rise to more spatially intermittent field structures with various flux, very similar to chaotic or turbulent patterns. Assuming that there is a perturbation $\delta \boldsymbol{E}$ at an initial time, then from the condition, $\nabla \cdot \boldsymbol{E}(\boldsymbol{r}, t)=\mathbf{0}$, we have $\boldsymbol{k} \cdot \delta \boldsymbol{E}=\mathbf{0}$. That is, longitudinal perturbation is not permitted. Hence the collapse is anisotropic. In addition, it was also found that the modulational instabilities with different growth rates in different directions occur and have the maximum growth rate in the $\boldsymbol{k}$-direction, perpendicular to wavevector $\boldsymbol{k}_{0}$ of the pump field. Therefore a pancake-like structure of the fields can be formed. Now let us assume that the nonlinear entity has a pancake form with a scale $\xi$ and radius $R \gg \xi$ and $\boldsymbol{E}$ has characteristic time-space scales $(\tau, \xi)$. For sub-sonic motion $(\xi / \tau<1)$, it is possible to neglect the term $\left(\partial^{2} / \partial \tau^{2}\right)$ in Eqs. (57) and (59). Then one has $n \sim-|\boldsymbol{E}|^{2}$ and $\boldsymbol{B}=i \frac{2}{3} \frac{\partial}{\partial \tau}\left(\boldsymbol{E} \times \boldsymbol{E}^{*}\right)$. Then we write the order of magnitudes of terms in Eq. (58):

$\frac{1}{\tau} \approx c_{1} \frac{\alpha}{\xi^{2}}+c_{2}|\boldsymbol{E}|^{2}+c_{3} \frac{|\boldsymbol{E}|^{2}}{\tau}$,

where we have used $\boldsymbol{E} \times \boldsymbol{B} \sim \frac{1}{\tau} \boldsymbol{E} \times\left(\boldsymbol{E} \times \boldsymbol{E}^{*}\right) \sim \frac{1}{\tau}\left(|\boldsymbol{E}|^{2} \boldsymbol{E}\right)$; $\left|c_{1}\right|,\left|c_{2}\right|$, and $\left|c_{3}\right|$ are constants of order unity; $\tau=\widetilde{t_{0}}-\widetilde{t}$, and $\widetilde{t_{0}}$ corresponds to $\bar{W}^{\mathrm{p}} \geq 1$, at which the perturbed fields become too strong for Eqs. (57), (58) and (65) to be valid. Thus we obtain from Eq. (83) for the sub-sonic collapse

$|\boldsymbol{E}|^{2} \sim \frac{1}{\tau}$

provided that the first and the last terms on the right side of Eq. (83) are balanced, which implies

$\alpha \frac{\tau}{\xi^{2}} \sim|\boldsymbol{E}|^{2}$

or in view of Eq. (84),

$\xi \sim \tau$.

In addition, multiplying (58) by $\boldsymbol{E}^{*}$ and subtracting from it the complex conjugate equation multiplied by $\boldsymbol{E}$, we get

$i \frac{\partial|\boldsymbol{E}|^{2}}{\partial \tau}+\nabla \cdot\left[\alpha\left(\nabla \times \boldsymbol{E}^{*}\right) \times \boldsymbol{E}-\alpha(\nabla \times \boldsymbol{E}) \times \boldsymbol{E}^{*}\right]=0 ;$

then integrating over all space yields the conservation of the plasmon number: $\int|\boldsymbol{E}|^{2} \mathrm{~d} \xi=$ const., which gives

$|\boldsymbol{E}|^{2} \xi \pi R^{2}=$ const., i.e.,

$|\boldsymbol{E}| \sqrt{\xi} R=$ const.

Therefore, a self-similar collapse solution can be asymptotically shown as

$|\boldsymbol{E}| \simeq \frac{1}{\sqrt{\tau}} F\left(\frac{\xi}{\tau}\right)$

with $R \sim$ const.,where $F$ is a function determined by the initial conditions. For supersonic motion $(\xi / \tau=$ const. $>1)$, it is possible to neglect the term $\left(\nabla^{2}\right)$ in Eqs. (57) and (59); and by similar considerations, it is proved that the solution (87) still is valid.

For the same reason discussed before (see Sect. 2.3), we may identify the $\xi_{\text {crit }}$ as the scale $\xi_{\text {coll }}$ to stop collapse, which corresponds to $\bar{W}^{\mathrm{p}} \sim 1$. The maximum growth rate $\gamma_{\text {coll,max }}$ by collapse motion could be obtained from the self-similar solution (87) as

$\gamma_{\text {coll,max }}^{-1}=\tau_{\text {coll }}=\tau_{\mathrm{M}}\left(\frac{\xi_{\text {crit }}}{\xi_{\mathrm{M}}}\right)$ 
where $\gamma_{\mathrm{M}}=\left(\tau_{\mathrm{M}}\right)^{-1}$ is maximum growth rate for modulational instabilities. In view of (77) and (79), Eq. (88) yields that

$\tau_{\text {coll }}=\frac{1}{\sqrt{2}}\left(\left|\boldsymbol{E}_{0}\right|^{2}\right)^{-2 / 3} \alpha^{1 / 3}$.

Now we can obtain from Eq. (59)

$B_{\max } \sim\left(\frac{\tau}{\xi}\right)^{2} \frac{1}{\tau_{\text {coll }}} \frac{2}{3}|\boldsymbol{E}|_{\text {coll }}^{2}$

with $\frac{\xi}{\tau}=$ const. $>1$. The collapse fields satisfy the condition of $\bar{W}_{\text {coll }}^{\mathrm{p}}=\frac{2}{3} \mu|\boldsymbol{E}|_{\text {coll }}^{2} \sim 1$, i.e., up to an uncertainty of order unity,

$B_{\max }^{2} \simeq 2 \frac{\left(\left|\boldsymbol{E}_{0}\right|^{2}\right)^{4 / 3}}{\alpha^{2 / 3}} \mu^{-2}$.

In dimensional unit for $(\delta \boldsymbol{B})^{2}$ (see Eq. (62)),

$\frac{\delta \boldsymbol{B}_{\max }^{2}}{8 \pi}=\frac{8}{9} \mu^{3} c^{2} \rho B_{\max }^{2}=\frac{16}{9} \mu c^{2} \rho \frac{\left(\left|\boldsymbol{E}_{0}\right|^{2}\right)^{4 / 3}}{\alpha^{2 / 3}}$,

where $\rho \simeq m_{\mathrm{i}} n_{\mathrm{e}}$ for a fully ionized hydrogen plasma. By virtue of condition (52) and the condition $\beta>>1$, the initial amplitude $\left|\boldsymbol{E}_{0}\right|^{2}$ of the pumping waves in dimensionless units is restricted by the relation

$\alpha^{-1} \ll\left|\boldsymbol{E}_{0}\right|^{2}<\mu^{-1}$.

It should be pointed that numerical simulations of the selfgenerated fields illustrate the tendency to self-similar collapse. The maximum value of the resulting magnetic fields, $\left(B_{\max }^{\mathrm{I}}\right)_{\mathrm{C}} \simeq$ 56.2 (with $\alpha=3.3 \times 10^{5}$ and $\left|\boldsymbol{E}_{0}\right|^{2}=6.2$ ), is observed (Li \& Ma 1994) in the numerical solution of Eqs. (57), (58) and (65). For sub-sonic motion, we have $\left(B_{\max }^{\mathrm{II}}\right)_{\mathrm{C}} \simeq 1.4 \times 10^{2}$ (with $\alpha=3.3 \times 10^{5}$ and $\left.\left|\boldsymbol{E}_{0}\right|^{2}=3.98\right)\left(\mathrm{Li} \&\right.$ Ma 1993), $\left(B_{\max }^{\mathrm{III}}\right)_{\mathrm{C}} \simeq$ $3.8 \times 10^{3}$ (with $\alpha=610$ and $\left|\boldsymbol{E}_{0}\right|^{2}=6$ ) (Liu \& Li 2000). In addition we get the magnetic field values of the self-similar solution from Eq. (90): $\left(B_{\max }^{\mathrm{I}}\right)_{\mathrm{S}} \simeq 130,\left(B_{\max }^{\mathrm{II}}\right)_{\mathrm{S}} \simeq 0.95 \times 10^{2}$ and $\left(B_{\max }^{\mathrm{III}}\right)_{\mathrm{S}} \simeq 1 \times 10^{3}$. This means that numerical solutions reach asymptotically the similar form (90) within a factor $\chi \leq 3$.

\subsection{Anomalous resistivity and magnetic viscosity}

The charged particles in the plasmas are scattered by the lowfrequency magnetic flux; the anomalous resistivity arises from such process. Anomalous resistivity from the low-frequency magnetic flux is a dc electric resistivity. For the self-generated flux with low-frequency, $v_{\mathrm{s}}<\omega / k \ll v_{T_{\mathrm{e}}}$, it follows the lowfrequency, i.e., dc conductivity from $(56)\left(i \omega \approx \gamma_{\mathrm{M}}\right)$

$\sigma_{\mathrm{dc}}=\frac{\omega_{\mathrm{pe}}^{2}}{4 \pi} \frac{\gamma_{\mathrm{M}}}{k_{\max }^{2} v_{T_{\mathrm{e}}}^{2}} ;$

or in view of (74) and (75) in dimensional units,

$\sigma_{\mathrm{dc}}=2^{-1 / 3} \frac{\omega_{\mathrm{pe}}}{8 \pi} \mu^{1 / 6}\left(\frac{c}{v_{T_{\mathrm{e}}}}\right)^{5 / 3}\left(\bar{W}_{0}^{\mathrm{p}}\right)^{-1 / 6}$.

Hence one has

$$
\begin{aligned}
\eta_{\text {dif }} & \equiv c^{2} /\left(4 \pi \sigma_{\mathrm{dc}}\right)=2^{4 / 3}\left(\bar{W}_{0}^{\mathrm{p}}\right)^{1 / 6} \mu^{-1 / 6}\left(\frac{v_{T_{\mathrm{e}}}}{c}\right)^{2 / 3} d_{\mathrm{e}} c \\
& =1.5 \times 10^{8}\left(\frac{\bar{W}_{0}^{\mathrm{p}}}{10^{-5}}\right)^{1 / 6} T_{0}^{5 / 6} n_{0}^{-1 / 2}\left(\frac{T_{\mathrm{e}}}{T_{0}}\right)^{5 / 6}\left(\frac{n_{\mathrm{e}}}{n_{0}}\right)^{-1 / 2},
\end{aligned}
$$

here $n_{0}\left(\mathrm{~cm}^{-3}\right)$ and $T_{0}(\mathrm{~K})$ are the fiducial density and temperature of interest in the problem.

The magnetic viscous force by the self-generated spatially intermittent flux is

$f_{i}^{\mathrm{m}}=\nabla_{j} t_{i j}^{\mathrm{m}}$,

where the viscous stress tensor is

$t_{i j}^{\mathrm{m}}=\left\langle\delta B_{i} \delta B_{j}-\frac{1}{2} \delta_{i j}(\delta \boldsymbol{B})^{2}\right\rangle / 4 \pi$.

The work done on the volume $\mathrm{d} \boldsymbol{r}$, per unit time, by the stress is

$-\frac{\partial t_{i j}^{\mathrm{m}}}{\partial x_{j}} v_{i} \mathrm{~d} \boldsymbol{r}$

which contributes to the heat energy due to viscous dissipation, resulting in the change of the entropy within the volume:

$\dot{S}=\int \frac{1}{T}\left(-\frac{\partial t_{i j}^{\mathrm{m}}}{\partial x_{j}} v_{i}\right) \mathrm{d} \boldsymbol{r}=\int t_{\mathrm{ij}}^{m} \frac{V_{i j}}{T} \mathrm{~d} \boldsymbol{r}$,

where

$V_{i j} \equiv \frac{1}{2}\left(\frac{\partial v_{i}}{\partial x_{j}}+\frac{\partial v_{j}}{\partial x_{i}}\right)$

Equation (98) corresponds to that linear relation of the "flow" $\left(t_{i j}^{\mathrm{m}}\right)$ and the "force" $\left(\frac{-V_{i j}}{T}\right)($ Lifshitz \& Pitaevskii 1981):

$t_{i j}^{\mathrm{m}}=\gamma_{i j ; l k} \frac{V_{l k}}{T}=\eta_{i j, l k} V_{l k}$

here $\eta_{i j ; l k}$ is kinetic coefficients. This equation above can be recast as

$t_{i j}^{\mathrm{m}}=\eta_{\mathrm{m}}\left(\frac{\partial v_{i}}{\partial x_{j}}+\frac{\partial v_{j}}{\partial x_{i}}-\frac{2}{3} \delta_{i j} \frac{\partial v_{k}}{\partial x_{k}}\right)$

with

$\eta_{i j ; l k}=\eta_{\mathrm{m}}\left(\delta_{i l} \delta_{j k}+\delta_{i k} \delta_{j l}-\frac{2}{3} \delta_{i j} \delta_{l k}\right)$,

where $\eta_{\mathrm{m}}$ is magnetic viscosity defined through the kinetic coefficient as $\eta_{\mathrm{m}}=\eta_{l k, l k}(l \neq k$, no summation over repeated $l k)$.

In many practical cases including the accretion disk and the Galaxy, the shear stress tensor $V_{l k}$ has the dominant $r \varphi$-component and then Eq. (99) becomes

$t_{i j}^{\mathrm{m}}=\eta_{i j, r \varphi} \frac{1}{2}\left(\frac{\partial v_{\varphi}}{\partial r}-\frac{v_{\varphi}}{r}\right)=\eta_{i j, r \varphi} \frac{1}{2} r \frac{\partial \Omega(r)}{\partial r}$.

Furthermore, it yields from Eqs. (97), (101) and (102)

$\frac{1}{4 \pi}\left|\left\langle\delta B_{\mathrm{r}} \delta B_{\varphi}\right\rangle\right|=\eta_{\mathrm{m}} \frac{1}{2} r\left|\frac{\partial \Omega(r)}{\partial r}\right|$.

For turbulent transverse plasmons, that is, turbulence dominated by the behavior of the transverse modes $\omega^{\mathrm{p}} \approx \omega_{\mathrm{pe}}+\frac{k^{2} c^{2}}{2 \omega_{\mathrm{p}}}$, the pumping waves are in various phases. In this circumstance, we may assume that self-generated magnetic fields by the transverse modes are statistically isotropic on the scales of interest:

$\frac{1}{3}\left\langle(\delta \boldsymbol{B})^{2}\right\rangle \approx\left\langle\delta B_{\mathrm{r}} \delta B_{\varphi}\right\rangle$ 
Up to an order of unity, one has $\left\langle(\delta \boldsymbol{B})^{2}\right\rangle \approx\left(\delta \boldsymbol{B}_{\max }^{2}\right)$; and then we obtain the magnetic kinematic viscosity $v_{\mathrm{m}}$ from Eqs. (103), (104) and (91):

$v_{\mathrm{m}} \equiv \eta_{\mathrm{m}} / \rho=\frac{64}{27} \mu c^{2} \frac{\left(\left|\boldsymbol{E}_{0}\right|^{2}\right)^{4 / 3}}{\alpha^{2 / 3}} \frac{1}{r\left|\frac{\partial \Omega(r)}{\partial r}\right|}$,

or, via the turbulence parameter of pumping waves, $\bar{W}_{0}^{\mathrm{p}}$, it can be read as

$$
\begin{aligned}
v_{\mathrm{m}}= & 7 \times 10^{-12} \frac{c^{2}}{r\left|\frac{\partial \Omega(r)}{\partial r}\right|} T_{0}^{2 / 3}\left(\frac{T_{\mathrm{e}}}{T_{0}}\right)^{2 / 3} \\
& \times\left(\frac{\bar{W}_{0}^{\mathrm{p}}}{10^{-5}}\right)^{4 / 3}\left(\mathrm{~cm}^{2} / \mathrm{s}\right) .
\end{aligned}
$$

In summary, we obtain the anomalous magnetic Prandtl number

$$
\begin{aligned}
P_{\mathrm{a}} \equiv \frac{v_{\mathrm{m}}}{\eta_{\text {dif }}}= & 4.7 \times 10^{-20}\left(\frac{\bar{W}_{0}^{\mathrm{p}}}{10^{-5}}\right)^{7 / 6} \frac{c^{2}}{r\left|\frac{\partial \Omega(r)}{\partial r}\right|} \\
& \times T_{0}^{-1 / 6}\left(\frac{T_{\mathrm{e}}}{T_{0}}\right)^{-1 / 6} n_{0}^{1 / 2}\left(\frac{n_{\mathrm{e}}}{n_{0}}\right)^{1 / 2},
\end{aligned}
$$

which is very different from the microscopic magnetic Prandtl number (see below). Note that $P_{\mathrm{a}}$ is not very sensitive to the temperature $T_{\mathrm{e}}$, and this is probably important in stability analyses of disks.

For a Keplerian rotation thin disk, $\Omega(r)=\Omega_{\mathrm{K}}=$ $\sqrt{G M} r^{-3 / 2}$, Eq. (103) becomes

$\frac{\delta \boldsymbol{B}_{\max }^{2}}{8 \pi}=\frac{9}{8} \eta_{\mathrm{m}} \Omega_{\mathrm{K}}$

and putting $\eta_{\mathrm{m}} \approx \eta_{\mathrm{t}}$ (see Eq. (4)) and $\Omega_{\mathrm{K}} H \approx c_{\mathrm{s}}$, we get formally

$$
\frac{\delta \boldsymbol{B}_{\max }^{2}}{8 \pi}=\frac{9}{8} \alpha_{\mathrm{t}} \rho c_{\mathrm{s}}^{2}=\frac{9}{8} \alpha_{\mathrm{t}} P .
$$

Or, by use of Eq. (91)

$$
\begin{aligned}
\alpha_{\mathrm{t}} & =\frac{128}{81} \mu \frac{c^{2}}{c_{\mathrm{s}}^{2}} \frac{\left(\left|\boldsymbol{E}_{0}\right|^{2}\right)^{4 / 3}}{\alpha^{2 / 3}} \\
& =0.17\left(\frac{T_{\mathrm{e}}}{3 \times 10^{7}}\right)^{-1 / 3}\left(\frac{\bar{W}_{0}^{\mathrm{p}}}{10^{-5}}\right)^{4 / 3}
\end{aligned}
$$

Furthermore we can obtain the lower limit of the parameter $\alpha_{\mathrm{t}}$ from the condition (80):

$\alpha_{\mathrm{t}} \gg \alpha_{\mathrm{L}} \equiv \frac{128}{27} \frac{3 v_{T_{\mathrm{e}}}^{2}}{c^{2}}=7 \times 10^{-2}\left(\frac{T_{\mathrm{e}}}{3 \times 10^{7}}\right)$.

When the pressure of the enhanced magnetic flux becomes comparable to the total pressure $P$ the classical $\alpha_{\mathrm{t}}$ parameter will be no longer small as compared to unity. Then the disk is unstable against clumping of matter and bulging of the flux; and the flux would be expelled from the disk, leading to decrease of the parameter $\alpha_{\mathrm{t}}$. This is the so-called buoyancy limitation. However, the buoyant evolution has to appeal to the properties of magnetohydrodynamics with the large scale flow and stratification in disks. This is an exceedingly difficult task.
Hence the role of magnetic buoyancy is still not completely determined (Heyvaerts 1991).

For comparison with the microscopic resistivity and viscosity, we may consult Spitzer (1962) for values of the dissipation parameters in a fully ionized hydrogen plasma, they are

$$
\begin{aligned}
\eta_{\mu} & =\left(\frac{e^{2}}{m_{\mathrm{e}} c^{2}}\right) c\left(\frac{v_{T_{\mathrm{e}}}}{c}\right)^{-3} \\
& =3.8 \times 10^{12} T_{0}^{-3 / 2}\left(\frac{T_{\mathrm{e}}}{T_{0}}\right)^{-3 / 2},\left(\mathrm{~cm}^{2} / \mathrm{s}\right) \\
v_{\mu} & =2.2 \times 10^{-16} \rho_{0}^{-1} T_{0}^{5 / 2}\left(\frac{\rho}{\rho_{0}}\right)^{-1}\left(\frac{T_{\mathrm{e}}}{T_{0}}\right)^{5 / 2}, \quad\left(\mathrm{~cm}^{2} / \mathrm{s}\right)
\end{aligned}
$$

with the microscopic magnetic Prandtl number,

$P_{\mu} \equiv \frac{v_{\mu}}{\eta_{\mu}}=5.8 \times 10^{-29} T_{0}^{4}\left(\frac{T_{\mathrm{e}}}{T_{0}}\right)^{4} \rho_{0}^{-1}\left(\frac{\rho}{\rho_{0}}\right)^{-1}$.

For accretion disks around a black hole $\left(T_{\mathrm{e}} \sim 3 \times 10^{7} \mathrm{~K}, \rho \sim\right.$ $10^{-8} \mathrm{~g} / \mathrm{cm}^{3}, M \sim 8 M_{\odot}, \Omega \sim 4 \times 10^{-6} \mathrm{~s}^{-1}$ at $\left.r \sim 9 \times 10^{14} \mathrm{~cm}\right)$ the $\eta_{\mu}$ has a value very much smaller than $\eta_{\text {dif }}: \frac{\eta_{\text {dif }}}{\eta_{\mu}} \sim 10^{5}$; and the magnetic kinematic viscosity $v_{\mathrm{m}}$ would result in a factor of more than $10^{8}$ amplification to microscopic viscosity $v_{\mu}$. In addition, if the buoyancy limitation is adopted, we find a universal range for $\alpha_{\mathrm{s}}, 0.01<\alpha_{\mathrm{t}} \leq 1$, which often has been assumed in previous models. But if the buoyancy limitation is not valid, it is possible, in principle, that $\alpha_{\mathrm{t}}>1$; in this case one has from Eq. (110):

$\bar{W}_{0}^{\mathrm{p}}>3.8 \times 10^{-5}\left(\frac{T_{\mathrm{e}}}{3 \times 10^{7}}\right)^{1 / 4}$.

\section{Conclusion}

The subject of this paper is the collapsing magnetic instability from transverse plasmons and its applications. We have dealt with the issue in two regimes: magnetohydrodynamics and plasma kinetics, operated in the problems of solar magnetic fields and astrophysical accretion disks, respectively, considering their related scales underlying their physical processes.

In the first case, starting from three-component fluid equations, it is shown that nonlinear development of the modulational instability results eventually in a self-similar collapse of the transverse field and the equilibrium between the ponderomotive and the Lorentz forces within the sheet gives rise to a spatially intermittent, collapsing magnetic flux. So the flux cells emerging at the surface become shredded and the flux thereby is contracted rather quickly to a small-scale of the order of $100 \mathrm{~km}$, with a field strength of about 1-2 kG.

In the second case, based on the Vlasov equations, the modulational instability of the self-generated magnetic fields is explored, showing the possible collapse of the magnetic flux. The resultant anomalous dc resistivity and anomalous magnetic viscosity in accretion disks are calculated. We find that the magnetic Prandtl number is very different from the microscopic one and a universal range for the $\alpha_{\mathrm{t}}: 0.01<\alpha_{\mathrm{t}} \leq 1$, if the buoyancy limitation is adopted.

Acknowledgements. The work was supported by the National Natural Science Foundation of China. 


\section{References}

Balbus, S. A., \& Hawley, J. F. 1998, Rev. Mod. Phys., 70, 1

Bel'kov, S. A., \& Tsytovich, V. N. 1979, JETP, 49, 656

Coroniti, F. V. 1981, ApJ, 244, 587

Eardley, D. N., \& Lightman, A. P. 1975, ApJ, 200, 187

Gorev, V. V., Kingsep, A. S., \& Rudakov, L. I. 1976, Radioph. Qu. Electron, 19, 486

Hakimov, F. K., \& Tsytovich, V. N. 1976, Sov. JETP, 70, 1785

Heyvaerts, J. 1991, in Structure and Emission Properties of Accretion Disks, ed. C. Bertout, et al. (Éditions Frontières, Singapore), 109

Kaplan, S. A., \& Tsytovich, V. N. 1973, Plasma Astrophysics (Pergamon, London), Sects. 8, 2

Li, X. Q. 1989, Astrophys. Space Sci., 153, 311

Li, X. Q., \& Ma, Y. H. 1993, A\&A, 270, 534

Li, X. Q., \& Ma, Y. H. 1994, Phys. Plasmas, 1, 3008

Li, X. Q., \& Wu, S. T. 1989, in Laboratory and Space Plasma, ed. H. Kihuch (Springer-Verlag, New York), 239

Li, X. Q., \& Zhang, H. 1997a, A\&A, 327, 333

Li, X. Q., \& Zhang, Z. D. 1997b, ApJ, 479, 1028

Li, X. Q., Song, M. T., Hu, F. M., \& Fang, C. 1997, A\&A, 320, 300

Li, X. Q., Zhang, H., \& Li, Q. B. 1995, A\&A, 304, 617

Lifshitz E. M., \& Pitaevskii L. P. 1981, Physical Kinetics (Pergamon Press), 27
Liu, S. Q., \& Li, X. Q. 2000, A\&A, 364,785

Lovelace, R. V. E., Wang, J. C. L., \& Sulkanen, M. E. 1987, ApJ, 315, 504

Parker, E. M. 1978, ApJ, 221, 368

Peckover, R. S., \& Weiss, N. O. 1972, Comp. Phys. Comm., 4, 339

Schramkowski, G. P., \& Torkelsson, U. 1996, A\&AR, 7, 55

Shapiro, V. D., \& Shevchenko, V. I. 1984, in ed. A. A. Galeev, \& R. N. Sudan, Basic plasma Physics II (North-Holland Physics Pub., Amsterdam), 172

Spitzer, L. 1962, Physics of Fully Ionized Gases (Wiley Inter-science, New York)

Spruit, H. C. 1979, Solar Phys., 61, 363

Stenflo, J. O. 1989, A\&AR, 1, 3

Stepinski, T. F. 1992, Icarus, 97, 130

Tagger, M., Falgarone, E., \& Shukurov, A. 1995, ApJ, 299, 940

Torkelsson, U. 1993, A\&A, 274, 675

Tsytovich, V. N. 1977, Theory of Turbulent Plasma, Consultants Bureau, New York, 44

Weiss, N. O. 1966, Proc. Royal Soc. London A 293, 310

Zahn, J. P. 1991, in Structure and Emission Properties of Accretion Disks, ed. C. Bertout, et al. (Éditions Frontières, Singapore), 87

Zhang, S. N., et al. 2000, Science, 287, 1239 